\title{
GRAPH SPECTRAL ANALYSIS OF VOXEL-WISE BRAIN GRAPHS FROM DIFFUSION-WEIGHTED MRI
}

\author{
Anjali Tarun ${ }^{1,2}$, David Abramian ${ }^{3,4}$, Hamid Behjat $^{5}$, Dimitri Van De Ville ${ }^{1,2}$ \\ ${ }^{1}$ Institute of Bioengineering, Ecole Polytechnique Federale de Lausanne (EPFL), Switzerland \\ 2 Department of Radiology and Medical Informatics, University of Geneva (UNIGE), Switzerland \\ ${ }^{3}$ Department of Biomedical Engineering, University of Linkping, Sweden \\ ${ }^{4}$ Center for Medical Image Science and Visualization, University of Linkping, Sweden \\ ${ }^{5}$ Department of Biomedical Engineering, Lund University, Lund, Sweden
}

\begin{abstract}
Non-invasive characterization of brain structure has been made possible by the introduction of magnetic resonance imaging (MRI). Graph modeling of structural connectivity has been useful, but is often limited to defining nodes as regions from a brain atlas. Here, we propose two methods for encoding structural connectivity in a huge brain graph at the voxel-level resolution (i.e., 850'000 voxels) based on diffusion tensor imaging (DTI) and the orientation density functions (ODF), respectively. The eigendecomposition of the brain graph's Laplacian operator is then showing highlyresolved eigenmodes that reflect distributed structural features which are in good correspondence with major white matter tracks. To investigate the intrinsic dimensionality of eigenspace across subjects, we used a Procrustes validation that characterizes inter-subject variability. We found that the ODF approach using 3-neighborhood captures the most information from the diffusion-weighted MRI. The proposed methods open a wide range of possibilities for new research avenues, especially in the field of graph signal processing applied to functional brain imaging.
\end{abstract}

Index Terms - brain graph, eigenmodes, diffusion tensor imaging, orientation density functions

\section{INTRODUCTION}

Diffusion-weighted magnetic resonance imaging (DW-MRI) allows for in vivo visualization of the diffusion of water molecules in neural fibers, thereby revealing the underlying brain structure. Conventionally, various signal reconstruction techniques as well as tractography algorithms $[1,2]$ are used to derive white matter streamlines that connect the different cortical and subcortical regions of the brain [3].

Viewed under the lens of graph theoretical approaches, distinct brain regions are usually represented as graph vertices. The graph edges and their associated weights encode the strength of the association among regions [4-9], either based on physical strength of the connections or the degree of their functional interplay. Whether it is a functional or structural graph, one often limits the study in region-wise analysis by averaging the neural activity or the number of fibers connecting brain regions that are specified by an a priori atlas. Two limitations can be seen in such analysis. First, the analysis is drastically affected by the choice of parcellation scheme. It also merely provides a macro-scale view of structural or functional connectivity. Second, the analysis on structural brain graphs varies depending on the algorithm employed to approximate the number of white matter (WM) tracts, and while they do reconstruct tractograms that are present, most of them also produce a significant amount of false positives [11].

In order to overcome these limitations, we propose to build a brain graph that is defined at a voxel-level resolution. Doing so would not require the use of any parcellation schemes and tractography algorithms, and instead built directly from the reconstructed diffusion data. We present two design schemes, one using DTI data and the other using ODF data. The ODF design is explored using two levels of neigborhood connectivity principle, namely, 3 and 5 connectivity in 3D. We show, for the first time, highly resolved human brain eigenmodes that recover major white matter tracks. The Laplacian spectra of the resulting graphs are compared to determine which design maximally encodes diffusion data.

\section{METHODS}

Similar to classical brain graphs, we define a brain graph as $\mathcal{G}_{v w}:=(\mathcal{V}, \mathbf{A})$, where $\mathcal{V}=\{1,2,3, \ldots, N\}$ is the set of $N$ nodes representing the brain voxels, and $\mathbf{A} \in N \times N$ is an adjacency matrix encoding the connection strength between neighboring voxels. The specific definition of neighboring voxels depends on the type of signal reconstruction being considered.

\subsection{DTI-based brain graph}

In DTI, the fiber orientation at a voxel is described by an ellipsoid defined by a real symmetric $3 \times 3$ matrix $\mathbf{T}$ called the diffusion tensor. The displacement probability of water molecules at a given time can then be approximated by a multivariate Gaussian with the diffusion tensor as the covariance 

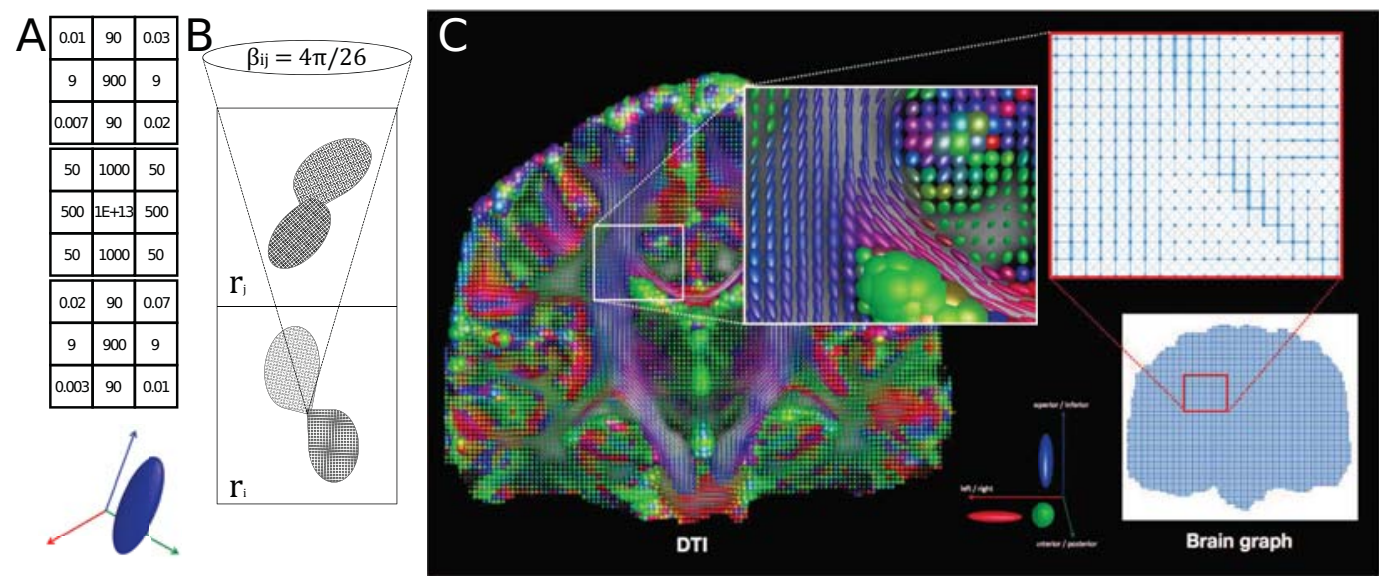

Fig. 1. (A) Filter coefficients obtained by discretizing the representative tensor shown; (B) adapts Itturia-Medina's original figure [10] illustrating the solid angle $\beta_{i j}$ around the vector $r_{i j}$, while (C) visualizes DTI data through ellipsoids, and the corresponding voxel-wise brain graph $(y=87)$.

matrix, given by [12]:

$$
P\left(\vec{r}_{i}, t_{i}\right)==\frac{1}{{\sqrt{4 \pi t_{i}}}^{3}|\mathbf{T}|} \exp \left(-\frac{{\overrightarrow{r_{i}}}^{T} \mathbf{T}^{-1} \overrightarrow{r_{i}}}{4 t}\right),
$$

where $\vec{r}_{i}$ and $t_{i}$ translate to the direction at a specific voxel and timepoint being considered. We used a cubic lattice of size $3 \times 3 \times 3$ Moore neighborhood to define the nodes of our brain graph, so that each node is connected up to a maximum of 26 nearest neighbors. The calculation of the equivalent weights requires a discretization step that guarantees a one-toone mapping between the (continuous) multivariate Gaussian model and the (discrete) weighting of vertices in the brain graph. This is done by assigning a cubic FIR filter in each voxel, defined as $h(k)$, and obtaining the filter coefficients that are equivalent to the diffusion ellipsoid that is modeled by Equation 1 . We specify $h(k)$ in terms of its transfer function as

$$
H(\mathbf{z})=\sum_{k} h(k) \mathbf{z}^{-k}
$$

where $\mathbf{z}^{k}$ is equivalent to $\prod_{i=1}^{i=M} z_{i}^{k_{i}}$, with $M=3$. The one to one mapping from the continuous domain to the discrete domain is achieved by matching equations 1 and 2 in their respective frequency domain representation. Fundamental signal processing concepts allow us to do the matching by noting that the Z-transform is essentially a discrete version of the Fourier transform (FT) if we set the real part of the complex variable to zero. Under the same frequency representation, we can expand in terms of their Taylor series approximation, and obtain the filter coefficients by matching the coefficients of the lowest order terms. The filter coefficients are normalized in each voxel and are multiplied with the corresponding fractional anisotropy (FA) in order to boost the structure of the graph. For each voxel $i$ in the brain, we encode the discrete counterpart of the diffusion ellipsoid onto the brain graph, noting that $A_{i, j}$ is given by the average of two coinciding filter coefficients coming from voxels $i$ and $j$. An example of a discretized ellipsoid is shown in Fig. 1(A) and a visualization of DTI ellipsoids and their corresponding voxel-wise brain graph for a representative coronal slice in Fig. 1(C).

\subsection{ODF-based brain graph}

We used an ODF-based weighting scheme that leverages previous work presented by Itturia-Medina $[10,13]$. Let $\mathcal{N}_{i}$ denote the set of vertices in $\mathcal{V}$ that are adjacent to vertex $i$. For any two vertices $i, j \in \mathcal{V}$, let $\vec{r}_{i j}$ denote the vector pointing from the center of vertex $i$ to the center of vertex $j$. Let $O_{i}(S)$ denote the ODF associated to voxel $r_{i}$, with its center of coordinate being the center of the voxel. We can then define

$$
p\left(i, \vec{r}_{i j}\right)=\int_{\beta_{i j}} O_{i}^{n}(S) d S
$$

as the probability of the nerve fibers being oriented along direction $\vec{r}_{i j}$. The variable $n$ is a positive integer that is a desired power factor to sharpen the ODFs, and $\beta_{i j}$ denotes a solid angle of $4 \pi / 98$ (for 5-neighborhood, $4 \pi / 26$ for 3 neighborhood) around $\vec{r}_{i j}$ subtended at the center of voxel $r_{i}$ (see Fig. 1(B)). Let $\left\{O_{i, k}\right\}_{k=1}^{N_{o}}$ denote the discrete samples of $O_{i}(S)$ along $N_{o}$ directions $\left\{\vec{r}_{k}\right\}_{k=1}^{N_{o}}$ from the center of the ODF. Thus, $p\left(i, \vec{r}_{i j}\right)$ can instead be approximated as a sum

$$
p\left(i, \vec{r}_{i j}\right) \approx \frac{4 \pi}{N_{o}} \sum_{k \in \mathcal{D}_{i, j}} O_{i, k}^{n}
$$

where $\mathcal{D}_{i, j}:\left\{k \mid \vec{r}_{k} \in \beta_{i j}\right\}$. The final weights of the brain graph are computed by taking into account the strength of the anisotropy of the nodes $r_{i}$ and $r_{j}$ and the ODF's orientation 


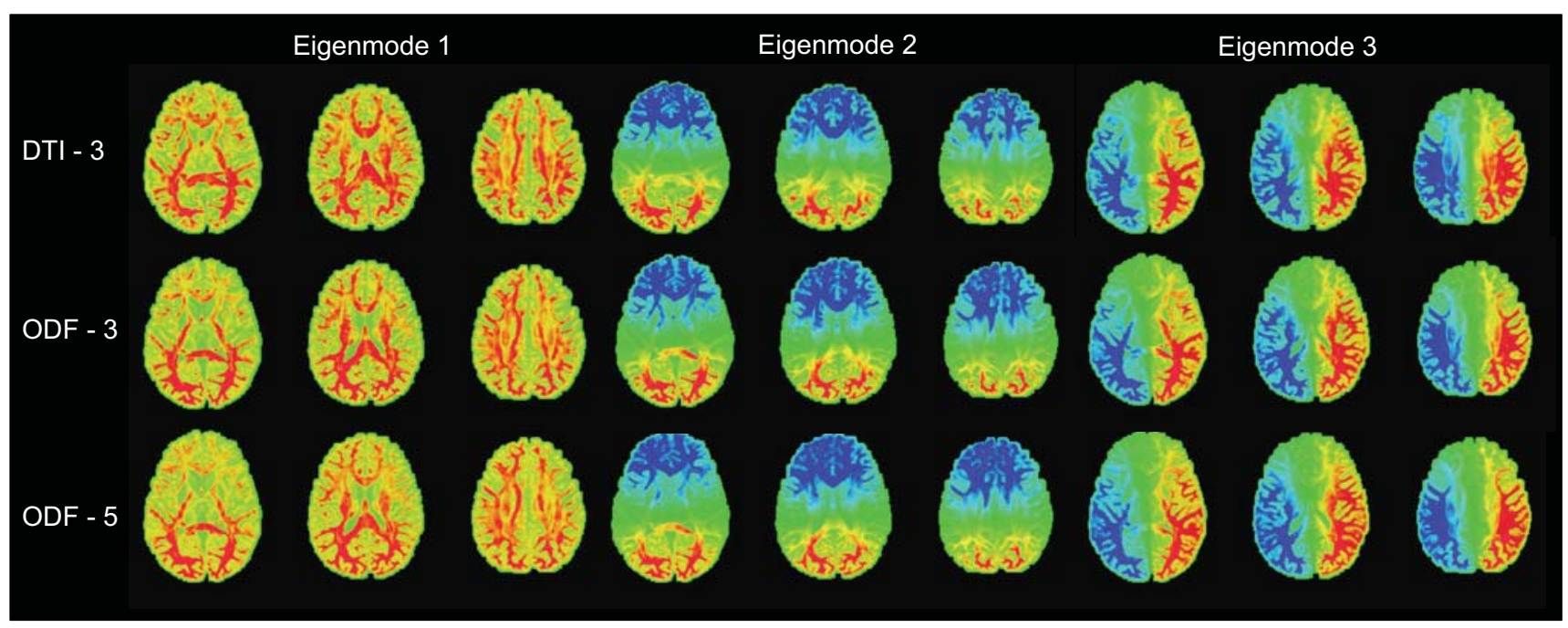

Fig. 2. Human brain eigenmodes of a representative subject corresponding to the top 3 lowest frequencies, produced by brain graphs constructed using 3-neighborhood DTI and 3 and 5-neighborhood ODF.

$p\left(i, \vec{r}_{i j}\right)$. Mathematically,

$$
A_{i, j}=P_{m a g}\left(r_{i}\right) p\left(i, \vec{r}_{i j}\right)+P_{m a g}\left(r_{j}\right) p\left(j, \vec{r}_{j i}\right)
$$

where $P_{\text {mag }}\left(r_{i}\right)$ is defined as

$$
P_{\text {mag }}\left(r_{i}\right)=\frac{Q A^{2}}{2 \max _{l \in \mathcal{N}_{k}} p\left(k, \vec{r}_{k l}\right)}
$$

where QA is the anisotropy index called the quantitative anisotropy that is originally defined by Yeh et. al [14], and the denominator is a normalization term for $p\left(i, \vec{r}_{i j}\right)$. Whereas Itturia-Medina [10] has defined a parameter based on tissue probability maps to describe the magnitude of the anatomical information, we propose to use QA, synonymous to FA in DTI. In particular, ODF provides the directionality (shape) of the diffusion, while the squared QA provides the magnitude (energy). By nature of the design of the ODF brain graph, we can choose to use a $3 \times 3 \times 3$ or a $5 \times 5 \times 5$ neighborhood, depending on the solid angle $\beta$ that we consider. The 5neighborhood intuitively offers a more resolved connectivity information, whereas the 3-neighborhood is more localized.

\section{RESULTS}

We constructed and evaluated three different brain graphs: (i) the 3-neighborhood DTI-based (DTI-3), (ii) the 3-neighborhood ODF-based (ODF-3) and (iii) the 5-neighborhood ODF-based (ODF-5) graph. A fourth graph with randomly assigned edge weights, using a Gaussian noise, is constructed and treated as a null for comparing and evaluating the brain graphs. The brain graphs are constructed within the native space where diffusion data were acquired, and it includes all voxels from all tissue types, i.e., gray matter (GM), white matter and the cerebrospinal fluid (CSF).

To capture the topology, we consider the graph Laplacian matrix in its symmetric normalized form $\mathbf{L}_{\mathrm{sym}}=$ $\mathbf{D}^{1 / 2} \mathbf{L} \mathbf{D}^{1 / 2}$. The eigendecomposition of $\mathbf{L}_{\mathbf{s y m}}$ leads to a complete set of orthonormal eigenvectors that span the graph spectral domain and of $N$ real, non-negative eigenvalues. The number of nodes typically range around 700-900 thousand for the whole brain graph, and as such, the dimension poses significant computational challenge. The present proofof-concept analysis was therefore confined to the first 1000 eigenvectors corresponding to the lowest spectral frequencies, estimated using the Krylov-Schur Algorithm [15].

The diffusion is inherently anisotropic in the WM in contrast to being isotropic in GM and CSF. This is reflected in Fig. 2 which illustrates the first three eigenmodes of the three brain graphs. Major WM structures clearly dominate the overall spatial pattern, indicating that the distinction between tissue types naturally arises from the assignment of the connectivity weights in the brain graph. The second and third eigenmodes show geometrical information of the head shape, dividing the brain into posterior and anterior, and left and right, respectively, while higher frequency eigenmodes show more spatial variability and more localized information.

By nature of the reconstruction models, ODFs are expected to recover more details, especially in crossings and branching fibers. However, as it is shown in Fig. 2, it is not easy to qualitatively pinpoint the differences of the approach through visual inspection. In order to characterize the differences in their topology, we look at the general trend of the eigenmodes in a population of 20 subjects from the Human Connectome Project (HCP) [16] database. 

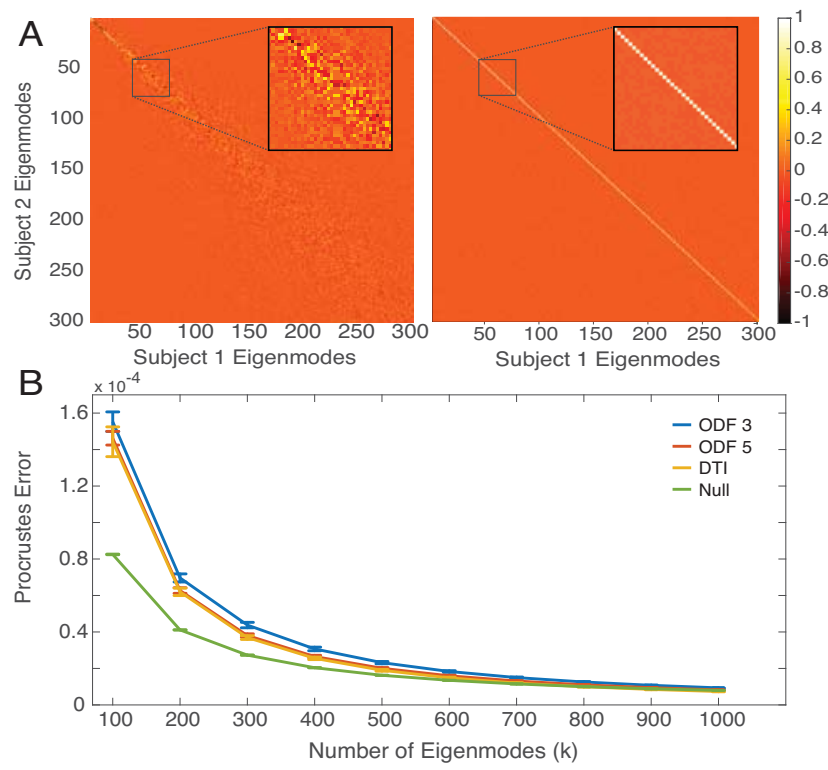

Fig. 3. (A) Cosine similarity of the first 300 eigenmodes of two representative subjects before (left) and after (right) Procrustes transform, where we see traces of flipped signs and unordered eigenmodes before applying the transformation. The curve in (B) summarizes the Procrustes error calculated by summing up off-diagonal values in all pair-wise cosine similarity matrices. The ODF- 3 shows the highest procrustes error, while the DTI and ODF-5 tied for second.

\subsection{Procrustes validation}

If the application calls for a group-level analysis, normalization using DARTEL [17] is suggested so that the deformation templates are specific to the group being analyzed. The eigendecomposition of the Laplacian returns subject-specific eigenmodes that are not necessarily in the same order with other subjects. To solve this, we used Procrustes transform [18] to flip the signs of the eigenmodes and re-order them accordingly. After an iterative Procrustes transformation, we obtain an averaged set of human brain eigenmodes representative of the population considered.

To examine the efficiency of the Procrustes transformation, we applied a cosine similarity measure to all pair-wise combination of subjects, see Fig. 3(A). Traces of flipped signs and unordered eigenmodes are observed before Procrustes transformation. Each set of eigenmodes contains subject-specific structural information, and while the Procrustes transformation matches similar eigenmodes, it cannot account for inherent inter-subject variability. Therefore, off-diagonal errors in the similarity matrix reflect these differences. We ran bootstrap methods to successively apply Procrustes transformation on a subset of 15 out of the total of 20 subjects. We examined the cosine similarity errors for increasing number of eigenmodes as is shown in Fig. 3(B). By summing up all off-diagonal values in the cosine similarity matrices, computed multiple times (with replacement) from all 20 subjects, we computed an error term, denoted Procrustes error. We found all four graphs showing a decreasing L-curve, having knee-points at around $k=300$ and reaching that of the null upon reaching higher $k$-values, suggesting that only the top 300 eigenmodes show relevant structure. The ODF-3 graph shows the highest procrustes error, while the DTI-3 and and ODF-5 graphs tied on the second spot, suggesting that the ODF-3 graph recovered the highest amount of information from the diffusion data.

\section{CONCLUSION}

Two design schemes for constructing voxel-vise brain graphs based on DTI and ODF were presented. The approach extends a previously proposed brain graph design limited to the GM [19,20], and a modified and improved versions of ItturiaMedina's ODF-based brain graph $[10,13]$. The brain graphs are constructed within the native diffusion space and have nodes covering the entire brain, including GM, WM and CSF. Thus, there are no coordinate transformation nor segmentation processes involved, making the brain graph more reliable and subject-specific. The decomposition of the Laplacian produced highly resolved human brain eigenmodes showing structural features that are in good correspondence with known major white matter bundles. Moreover, through a Procrustes validation scheme that is able to reflect inter-subject differences, we found that the 3-neighborhood ODF-based brain graph is better than the DTI-based owing to the fact that DTI is a much simpler model making it unable to reconstruct fiber crossings and branching patterns. Furthermore, we surmise that although the 3-neighborhood and 5-neighborhood ODF come from the same reconstruction method, the use of a higher-neighborhood scheme reduces the amount of information captured due to increased complexity and the possible inclusion of distant connections.

From a neuroscience perspective, the introduction of voxel-wise brain graphs opens a wide-range of new possibilities for the study of the brain. In the structural perspective alone, brain eigenmodes have been found as an effective biomarker for distinguishing healthy and diseased [8]. Moreover, brain eigenmodes have also been introduced as a building block for the human connectome [9] and cortical neural activity can be decomposed into frequency-specific modes. Similar to this, our method has the potential to extend the exploration not only in the cortex, but in the whole brain at a very high resolution. With the rising use of graph signal processing (GSP) in the field [5-7], this approach can propel studies aiming to understand how the dynamics of neural processes relate to the underlying fixed anatomical structure. The key is to define the functional data as signals residing in the voxel-wise brain grid, so that various GSP operations (e.g., filtering, signal inpainting) can then be tailored and explored according to the research question. 


\section{REFERENCES}

[1] T. E. J. Behrens, H. Johansen Berg, S. Jbabdi, M. F. S. Rushworth, and M. W. Woolrich, "Probabilistic diffusion tractography with multiple fibre orientations: What can we gain?," NeuroImage, vol. 34, no. 1, pp. $144-$ $155,2007$.

[2] H. Zhang, T. Schneider, C. A. Wheeler-Kingshott, and D. C. Alexander, "NODDI: Practical in vivo neurite orientation dispersion and density imaging of the human brain," NeuroImage, vol. 61, no. 4, pp. 1000 - 1016, 2012.

[3] S. Jbabdi, S. N. Sotiropoulos, S. N. Haber, D. C. Van Essen, and T. E. Behrens, "Measuring macroscopic brain connections in vivo," Nature Neuroscience, vol. 18, pp. $1546-1555,2015$.

[4] D. Van De Ville, R. Demesmaeker, and M. G. Preti, "When Slepian meets Fiedler: Putting a focus on the graph spectrum," IEEE Signal Processing Letters, vol. 24, pp. 1001-1004, 2017.

[5] T. A. W Bolton, Y. Farouj, S. Obertino, and D. Van De Ville, "Graph slepians to strike a balance between local and global network interactions: Application to functional brain imaging," 2018 IEEE 15th International Symposium on Biomedical Imaging, pp. 12391243, 2018.

[6] W. Huang, L. Goldsberry, N. F. Wymbs, S. T. Grafton, D. Bassett, and A. Ribeiro, "Graph frequency analysis of brain signals," IEEE J. Sel. Topic. Signal Process, vol. 10, no. 7, pp. $1189-1203,2016$.

[7] W. Huang, T. A. W. Bolton, J. Medaglia, D. Bassett, A. Ribeiro, and D. Van De Ville, "A graph signal processing perspective on functional brain imaging," Proceedings of the IEEE, vol. 106, no. 5, pp. 868-885, May 2018.

[8] M. B. Wang, J. P. Owen, P. Mukherjee, and A. Raj, "Brain network eigenmodes provide a robust and compact representation of the structural connectome in health and disease," PLOS Computational Biology, vol. 13, no. 6, pp. e1005550, 2017.

[9] S. Atasoy, I. Donnelly, and J. Pearson, "Human brain networks function in connectome-specific harmonic waves," Nature communications, vol. 7, 2016.

[10] Y Iturria-Medina, E. J. Canales-Rodriguez, L. MelieGarcia, P. A. Valdes-Hernandez, E Martinez-Montes, Y Alemán-Gómez, and JM Sánchez-Bornot, "Characterizing brain anatomical connections using diffusion weighted MRI and graph theory," Neuroimage, vol. 36, no. 3, pp. 645-660, 2007.
[11] K. H. Maier-Hein, P. F. Neher, J. C. Houde, M. A. Côté, E. Garyfallidis, J. Zhong, M. Chamberland, F. C. Yeh, Y. C. Lin, and Q. Ji et.al., "The challenge of mapping the human connectome based on diffusion tractography," Nature communications, vol. 8, no. 1, pp. 1349, 2017.

[12] A. L. Alexander, J. E. Lee, M. Lazar, and A. S. Field, "Diffusion tensor imaging of the brain," Neurotherapeutics, vol. 4, no. 3, pp. 316-329, 2007.

[13] S. N. Sotiropoulos, L. Bai, P. S. Morgan, C. S. Constantinescu, and C. R. Tench, "Brain tractography using qball imaging and graph theory: Improved connectivities through fibre crossings via a model-based approach," Neuroimage, vol. 49, no. 3, pp. 2444-2456, 2010.

[14] F. C. Yeh, V. J. Wedeen, and W. Y. I. Tseng, "Generalized q-sampling imaging," IEEE transactions on medical imaging, vol. 29, no. 9, pp. 1626-1635, 2010.

[15] G. W. Stewart, "A krylov-schur algorithm for large eigenproblems.," SIAM Journal of Matrix Analysis and Applications, vol. 23, no. 3, pp. 601-614, 2001.

[16] D. Van Essen, S. M. Smith, D. M. Barch, T. E. J. Behrens, E. Yacoub, and K. Ugurbil, "The WU-Minn human connectome project: An overview," Neuroimage, pp. 62-79, 2013.

[17] J. Ashburner, "A fast diffeomorphic image registration algorithm," NeuroImage, vol. 38, no. 1, pp. 95 - 113, 2007.

[18] C. Goodall, "Procrustes methods in the statistical analysis of shape," Journal of the Royal Statistical Society. Series B (Methodological), vol. 53, no. 2, pp. 285 - 339, 1991.

[19] H. Behjat, N. Leonardi, and D. Van De Ville, "Statistical parametric mapping of functional MRI data using wavelets adapted to the cerebral cortex," in Proc. IEEE Int. Symp. Biomed. Imaging, 2013, pp. 1070-1073.

[20] H. Behjat, N. Leonardi, L. Sornmo, and D. Van De Ville, "Anatomically-adapted graph wavelets for improved group-level fMRI activation mapping," $\mathrm{Neu}$ roImage, vol. 123, pp. 185 - 199, 2015. 